\title{
Uncovering bioactive compounds and potential mechanisms of Jieduan-Niwan Formula against liver failure
}

Chongyang Ma ( $\sim$ machongyang@live.com )

Capital Medical University

Qiuyun Zhang

Capital Medical University

Yuan Gao

Capital Medical University

Lianyin Gao

Capital Medical University

Xiaoyi Wei

Capital Medical University

Jiajun Liang

Capital Medical University

Wenlong Yang

Capital Medical University

Yuqiong Du

Capital Medical University

\section{Research}

Keywords:

Posted Date: July 24th, 2020

DOI: https://doi.org/10.21203/rs.3.rs-45023/v1

License: (1) (1) This work is licensed under a Creative Commons Attribution 4.0 International License.

Read Full License 


\section{Abstract \\ Background}

Liver failure is considered as the inability of the liver to perform its normal synthetic and metabolic function. Our previous data showed that Jieduan-Niwan formula (JDNW) induced liver failure, while its underlying mechanism remains unknown.

\section{Methods}

In the present study, we performed a network pharmacology to analyze the bioactive ingredients and related targets. Compound and target data of JDNW formula were retrieved from the Traditional Chinese Medicine Systems Pharmacology Database (TCMSP), and targets related to liver diseases were obtained from DisGeNET database. Information of protein protein interaction were retrieved from STRING database. Network construction and degree calculation were used Cytoscape software. Metascape webserver was used for GO and KEGG pathway enrichment. A microarray data series (GSE72081) was obtained from Gene Expression Omnibus database and analyzed using R project and Connectivity Map. Ledock was used for molecular docking.

\section{Results}

A total of 114 unduplicated active ingredients were identified after ADME screening. After construction of a target-liver disease network, 28 targets were identified for anti-liver failure effect. These targets were significantly enriched in fluid shear stress and atherosclerosis, pathways in cancer, IL-17 signaling pathway, HIF-1 signaling pathway, insulin resistance, toxoplasmosis, prostate cancer, microRNAs in cancer, NF-kappa B signaling pathway, Chemical carcinogenesis, VEGF signaling pathway and complement and coagulation cascades pathways. After analyzing a public microarray data of quercetin, we found biological action of some NFKB inhibitors was similar to quercetin. Molecular docking study showed that quercetin possessed a capability to bind on IKK $\beta$ and inhibited NFKB activation.

\section{Conclusions}

JDNW formula treated liver failure via multiple targets and multiple pathways, including NFKB signal. Quercetin was identified as a key bioactive ingredient of JDNW formula and its anti-liver failure effect may involve in NFKB inhibition.

\section{Background}

The liver is the biggest internal organ in human body and plays an important role in multiple physiological processes, such as metabolism of glucose and lipid, detoxification of various metabolites, 
synthesis and secretion of functional proteins, production of biochemicals necessary for digestion and activation of immune response. Liver failure is considered as the inability of the liver to perform its normal synthetic and metabolic function as part of normal physiology[1]. According data from 11 representative hospitals in China, about $5 \%$ of liver diseases patients suffered liver failure[2]. Liver failure may occur acutely without any underlying liver disease (acute liver failure, ALF), as an acute decompensation of chronic liver disease ('acute-on-chronic' liver failure, ACLF) or as a chronic decompensation of end-stage liver disease. In addition to the central liver failure, all the types are characterized by manifestations of systemic inflammation and end-organ dysfunctions, of which the most important are disturbances of circulation, renal and cerebral functions. Indeed, despite progression of clinical treatments, all the types of liver failure are still associated with increased mortality and morbidity and clinical management of liver failure remains challenging.

Traditional Chinese Medicine (TCM) therapies are widely used to treat liver disease in China and other Asian countries for their ability to protect hepatocytes, suppress hepatic inflammation, reduce liver fibrosis[3]. Jieduan-Niwan (JDNW) formula is widely regarded as the representative formula against ACLF, which is first created by Prof. Ying Qian. It is composed of ten herbs, including Phyllanthus urinaria (PU), Radix rehmanniae (RR), Panax Notoginseng (PN), Lysimachiae Herba (LH), Hedysarum Multijugum Maxim (HMM), Viscum Angulatum Heyne (VAH), Trichosanthes Kirilowii Maxim (TKM), Aconiti Lateralis Radix Praeparata (ALRP), Curcumae Rhizoma (CR) and Radix Salviae (RS). Our previous experiments showed that JDNW formula prolonged survival time, reversed liver tissue damage, reduced systemic inflammation and inhibited liver mitochondrial apoptosis in animal model of ACLF[4-6]. To further investigating the underlying mechanism of JDNW formula, a network-based strategy was used.

Network-based strategies hold great promise in supporting such transition from the "single-gene-based" paradigm to a "network-based" paradigm for drug discovery in many diseases, such as cancer[7], rheumatoid arthritis[8] and coronary heart disease[9]. Network pharmacology is also successfully applied in discovering biological mechanisms of Chinese medicine on liver diseases including liver fibrosis[10], non-alcoholic liver disease[11] and drug-induced liver injury[12]. In the present research, we used a network pharmacology-based strategy to identify bioactive ingredients in JDNW formula and predict its liver failure-related targets. We found biological processes and KEGG signaling pathways of JDNW formula against liver failure. Based on topological features, important ingredients treating liver failure were identified and further investigated via pharmaceutical informatics analysis.

\section{Methods}

\section{Identification of active ingredients}

The chemical ingredients of all the ten herbs that constitute JDNW formula was obtained from the Bioinformatics Analysis Tool for Molecular mechANism of Traditional Chinese Medicine (BATMAN-TCM) database[13] and the Traditional Chinese Medicine System Pharmacology (TCMSP) database[14]. Active ingredients were screened by setting $\mathrm{OB} \geq 30 \%, \mathrm{CACO}-2 \geq 0$ and $\mathrm{DL} \geq 0.18$ as the threshold. 
Furthermore, text mining was applied to gather the ingredients in plasma after oral administration of JDNW formula. The ten different herbs, PU, RR, PN, LH, HMM, VAH, TKM, ALRP, CR and RS contributed 6, $3,7,8,18,7,14,11,3$ and 57 active ingredients, respectively. In total, 114 unduplicated active ingredients were identified.

\section{Screening of drug-disease targets}

Targets of active ingredients were obtained from TCMSP database. DisGeNET database[15] was searched to identify liver diseases-related targets using some key words, including liver failure, liver cirrhosis, fatty liver, liver cancer and hepatitis. And gene names were uniformed by the UniProt Knowledgebase. The above targets were cross-referenced to identify the relationship between the liver disease-related target and the potential target of JDNW formula.

\section{Network construction and analysis}

All network was constructed using Cytoscape software (Version 3.2.1). Firstly, we constructed an interaction network for the identified active ingredients and their targets. Secondly, to explore the relationship between JDNW targets and liver diseases, a target-disease network was constructed containing five kinds of liver diseases, such as liver failure, liver cirrhosis, fatty liver, liver cancer and hepatitis. At last, a network containing liver failure-related targets and their corresponding active ingredients was constructed. The topological importance of each node in the network was assessed by calculating topological parameter, 'Degree centrality (DC)' with NetworkAnalyzer tool.

\section{Gene enrichment analysis and protein-protein interaction network}

The Metascape (http://metascape.org/)[16] was used for GO analysis, KEGG pathway analysis. A FDR pvalue $\leq 0.05$ was taken to declare a significant enrichment. String database was used for protein-protein interaction (PPI) network construction. After visualize PPI network, a MCODE based identification of subPPI network was performed automatically.

\section{Public Data Collection}

The Gene Expression Omnibus (GEO, http://www.ncbi.nlm.nih.gov/geo) is a public functional genomics data repository of high-throughput gene expression, chip, and microarray data. We downloaded the data series GSE72081 from the GEO database, which contains samples of the primary mouse hepatocytes (PMH) treated with DMSO and quercetin for $24 \mathrm{~h}$. All samples were processed on the GPL18615 Affymetrix Mouse Genome 430 2.0 Array. The probes were represented using the corresponding gene symbol according to the annotation information in the platform.

\section{Identification of DEGs}

The microarray data were preprocessed using RMA with the oligo and limma package in Bioconductor (v3.7; http://www.bioconductor.org/). Background correction, normalization, and calculation of 
expression were all included in the preprocessing stage. Microarray data probe IDs were transformed into gene symbols with the Bioconductor Annotation Data software packages. When several probes mapped to the same gene symbol, the mean value was set as the final expression value of this gene. $P$ values were acquired using the unpaired Student's t-test provided by the limma package and were adjusted using the Benjamin-Hochberg (BH) method. The thresholds for identifying DEGs were set as adjusted (adj.) $\mathrm{P}<$ 0.05 and $\mid \log 2$ fold change $(F C) \mid>1$. Hierarchical clustering analysis of the DEGs was then performed and visualized using g-plots as implemented in the $\mathrm{R}$ package.

\section{CMAP matching with existing compounds}

The latest Connectivity Map (CMap) CLUE web application (https://clue.io/cmap)[17] was used to further investigate the relationship between the effectiveness of quercetin on treating $\mathrm{PMH}$ and to explore its pharmacology and mechanism. The enrichment value in CMAP represented a similarity in gene mapping. A value close to 1 indicated a positive relationship between two drug molecules, which suggested that quercetin exhibited similar curative properties to the existing compounds.

\section{Docking analysis}

The interaction between NFKB and quercetin was evaluated by protein-ligand docking method by Ledock program as reported earlier, which had $80.8 \%$ accuracy for the best poses[18]. Following which, the protein was prepared and hydrogen atoms were added to the crystal structure by LePro tool. LeDock program is based on a combination of simulated annealing and evolutionary optimization of the ligand pose and its rotatable bonds (http://lephar.com). To investigate the docking poses, LeDock score function was used. It uses a physics/knowledge hybrid scoring scheme. The chemical structure of quercetin (CID: 5280343) was obtained from the pubchem compound database;

http://www.ncbi.nlm.nih.gov/pccompound. The structure of Human I kappa B Kinase beta with bound inhibitors (4kik) was recovered from the Protein Data Bank: http://www.pdb.org and was used as the starting point for examining the potential mode of binding of quercetin. Ligand was considered as a rigid body and the receptor was considered as flexible. The results were evaluated and sorted on the basis of predicted binding energy.

\section{Results}

\section{Screening Active ingredients and related targets of JDNW formula}

After screening was performed for the $\mathrm{OB}, \mathrm{CACO}-2$ and $\mathrm{DL}, 114$ unduplicated compounds were recognized as active ingredients of JDNW formula, including 6 PUs, 3 RRs, 7 PNs, 8 LHs, 18 HMMs, 7 VAHs, 14 TKMs, 11 ALRPs, 3 CRs and 57 RSs. The name, CAS code, and calculation parameter of these ingredients were shown in Table S1. Next, 154 unduplicated targets were recognized as potential targets of JDNW formula, indicating a multiple effect phenomenon. The name of these targets were shown in Table S2. 


\section{Ingredient-Target and Target-disease Network Constructions}

To further understand the complex interactions between the ingredients and their corresponding targets at a system level, we constructed an ingredient-target network based on the active ingredients of JDNW formula and their potential targets, which contained 254 nodes and 2191 edges (Figure 1a). Based on the relationship between targets and their related liver diseases, a target-disease network was also constructed, which contains 82 nodes and 165 edges (Figure 1b). In detail, the number of liver failurerelated targets was 28 , indicating a potential therapeutical effect of JDNW formula against liver failure. Besides, JDNW formula was also predicted to treat liver cirrhosis (50 targets), liver cancer (31 targets), fatty liver (31 targets) and hepatitis (25 targets).

\section{GO and KEGG enrichment of ingredient targets}

Using Metascape platform, we correlated biological process (BP) annotation with ingredient targets and grouped similar, redundant, and heterogeneous annotation terms into annotation groups according to the co-association of genes in different annotation terms. Terms with $\mathrm{P}<0.01$ and a minimum count of three were collected and grouped into clusters based on their membership similarities. As shown in Supplementary Figure $1 \mathrm{a}$ and $\mathrm{b}$, targets of JDNW formula were mainly enriched in cellular response to nitrogen compound, neurotransmitter receptor activity, response to toxic substance, chemical synaptic transmission, blood circulation, cellular response to organic cyclic compound, response to xenobiotic stimulus, regulation of neurotransmitter levels, positive regulation of MAPK cascade, response to ammonium ion, reactive oxygen species metabolic process, regulation of ion transport, response to nutrient levels, positive regulation of cellular component movement, response to steroid hormone, response to oxygen levels, regulation of body fluid levels, regulation of secretion, regulation of blood circulation and regulation of growth. As shown in Supplementary Figure $1 \mathrm{c}$ and d, these targets were enriched in neuroactive ligand-receptor interaction, fluid shear stress and atherosclerosis, pathway in cancer, CGMP-OKG signaling pathway, IL-17 signaling pathway, PI3K-Akt signaling pathway, retrograde endocannabinoid signaling, serotonergic synapse, endocrine resistance, dopaminergic synapse, cholinergic synapse, gap junction, drug metabolism-cytochrome P450, malaria, thyroid hormone signaling pathway, progesterone-mediated oocyte maturation, amyotrophic lateral sclerosis, regulation of lipolysis in adipocytes, prion diseases and platelet activation.

\section{Key ingredients against liver failure identification}

After annotated related-liver diseases of ingredients targets, we constructed a compound-liver failurerelated target interaction network, which contained 130 nodes and 578 edges (Figure 2). After calculating degree values of each nodes, we identified quercetin (degree 24), luteolin (degree 16), kaempferol (degree 16), tanshinone IIA (degree 11), beta-sitosterol (degree 10), isorhamnetin (degree 9), Stigmasterol (degree 9), acacetin (degree 9), dihydrotanshinone区(degree 9) and 2-isopropyl-8-methylphenanthrene-3,4-dione (degree 9). Compounds more than twice the median of degree values were identified as important ingredients of JDNW formula against liver failure, including quercetin, luteolin, kaempferol. 
KEGG enrichment and Protein-protein interaction analysis on intersecting targets of JDNW and liver failure

As shown in Figure $3 \mathrm{a}$ and $\mathrm{b}$, intersecting targets of JDNW and liver failure were enriched in fluid shear stress and atherosclerosis, pathways in cancer, IL-17 signaling pathway, HIF-1 signaling pathway, insulin resistance, toxoplasmosis, prostate cancer, microRNAs in cancer, NF-kappa B signaling pathway, Chemical carcinogenesis, VEGF signaling pathway and complement and coagulation cascades pathways. A PPI network containing 28 nodes and 182 edges was constructed (Figure $3 \mathrm{C}$ ). Sorted by degree value, top 10 nodes were TP53 (degree 24), VEGFA (degree 22), IL6 (degree 22), PTGS2 (degree 22), TNF (degree 21), MAPK8 (degree 20), IL1B (degree 17), CCL2 (degree 16), NOS3 (degree 15), PPARG (degree 15). Of note, some hub nodes were parts of NF-kappa B signaling pathway, such as PTGS2, TNF, IL6 and IL1B.

\section{Identification of the DEGs related to quercetin treatment}

One quercetin treated PMHs gene expression profile were downloaded from the NCBI GEO database. Afterwards, the gene expression data was normalized and DEGs were identified with the limma $R$ package $\left(P<0.05\right.$ and $\left.\left|\log _{2} F C\right|>1\right)$, and the results were shown in Figure 4 a and b. We screened out 614 DEGs, including 276 DEGs downregulated and 338 DEGs upregulated. Functional enrichment showed that quercetin involved in apoptotic process, oxidation-reduction process, immune system process and lipid and glucose metabolic process.

\section{Identification of drugs with similar effects by CMAP analysis and molecular docking}

The differentially expressed genes in PMH cells treated with quercetin were analyzed in the CMAP database. Connectivity scores close to 100 represent a high degree of positive correlation between the query signature and the reference profile in the CMAP database, which was derived from a specific chemical perturbation. Thus, the query compound may confer similar physiological effects on the cell as the matched chemical substance. In Figure 5 a, the top-ranking chemicals associated with anti-liver failure effect are listed. In CMAP analysis, we found biological action of some NFKB inhibitors was similar to quercetin, including parthenolide (Score:98.45), manumycin-a (Score:92.60), pyrrolidinedithiocarbamate (Score:92.12).

In order to examine the mode of binding between quercetin and potential IKK $\beta$ molecular domains, we carried out a docking model. The observed docking score for quercetin with IKK $\beta$ (PDB ID: 4KIK) was $-7.21 \mathrm{kcal} / \mathrm{mol}$. Molecular docking visualization showed that quercetin binds to various hydrophobic active site consisting of Glu214, Cys114, Phe219, Gly218, Lys428, GIn432 and Gly431 with hydrogen bonding to Arg220, Arg105, Arg427 and Try571 (Figure 5 b and c).

\section{Discussion}


JDNW formula is used to treat liver diseases clinically. According to traditional knowledge, some herbs in JDNW formula were used treating liver diseases. Especially, aqueous extract from Phyllanthus urinaria was found potential therapeutic effect on liver injury, hepatitis B and liver cancer[19]. Indeed, the relationship between JDNW formula and liver diseases mentioned above remains unknown. Future studies should discuss therapeutic potential against these diseases before clinical usage. Our previously published data showed that JDNW formula protected liver tissue against human serum albumin (HSA) combined with D-galactosamine (D-GaIN) and lipopolysaccharide (LPS)-induced liver failure, decreased mortality and inhibited hepatocyte apoptosis[20]. Therefore, the present study aimed at understanding the biological mechanisms of JDNW formula against liver failure and identification of important ingredients of this formula.

Our data showed that 102 ingredients were related to liver failure via their targets. Among these, some ingredients overlaps in different herbs. For example, stigmasterol was recognized as active ingredients in TKM, PN, RR and PU, while $\beta$-sitosterol was recognized in VAH, HMM, PN and PU. Recent research showed that stigmasterol inhibited LPS-induced acute liver injury with a dose-dependent manner[21]. $\beta$ Sitosterol was mentioned to decrease hepatofibrosis, inhibit the oxidation and inflammation against LPS/D-GaIN -induced acute hepatic failure and protect against CCI4-induced hepatotoxicity in animal models[22, 23].

Sorted by topological feature of ingredient-liver failure related target network, three compounds were identified as important ingredients of JDNW formula, including quercetin, luteolin, kaempferol. Previous research showed that quercetin (50 mg/kg, i.p.) alleviated D-galactosamine/lipopolysaccharide-induced liver failure in rats[24, 25]. More importantly, a phase I dose escalation study demonstrated quercetin safety (up to $5 \mathrm{~g}$ daily), indicating good hepatoprotective effects and potential as a promising therapeutic agent for liver failure in clinical application[26]. Luteolin (100 mg/kg, p.o.) was also confirmed by others' studies to have anti-oxidative, anti-inflammatory and anti-ER stress properties in response to acetaminophen-induced liver failure in mice[27, 28]. Keampferol and tanshinone IIA were supported against liver failure by literature[29,30]. No evidence about other ingredients against liver failure was reported. More importantly, experimental studies confirmed that Quercetin exists in five herbs of JDNW formula, including PU[31], HMM[32], LH[33], PN[34] and TKM[35] Therefore, we choose quercetin as a key ingredient of JDNW formula for further research and hoped quercetin could be a quality marker related to efficacy of JDNW formula. Further experiments should be carried out to make sure the effect of quercetin on liver failure and the role in JDNW formula.

According to studies on pharmacology, NFKB inhibitors may be potential antioxidants which suppress production of NFKB activators like ROS, or decrease proteasome and protease activities thereby inhibit NFKB P65/P50 complex, or inhibit IkBa phosphorylation, ubiquitination or degradation[36, 37]. As a phytochemical compound, quercetin was found to exhibit functions against production of free radicals and secretion of proinflammatory cytokines concurrent with activation of the NFKB pathway [38, 39]. In the present study, following predicting quercetin as a NFKB inhibitor, we discussed the unknown mechanism by which quercetin regulate NFKB activation. Evidence showed that the mechanism of 
quercetin on NFKB activation was complex. Quercetin could partially inhibit a ROS producer, COX-2 enzyme by binding to subunit $A$, and regulate the activator of NFKB and its downstream effects[40]. Our recently published data indicated that quercetin inhibited NFKB signal activation in an in vitro model of liver failure without identification of its direct targets[41]. In the present study, we demonstrated for the first time that quercetin acted as a potential inhibitor of IKKß, the key enzyme that regulates NFKB cascades and thus suppresses its downstream proinflammatory effects. Protein-ligand docking and molecular dynamics simulation were carried out for understanding the property of quercetin in structural and the microscopic level. Unlike results from studies mentioned above, quercetin was found accelerate wound closure, reduce immune cell infiltration and proinflammatory cytokines production by suppressing MAPK pathway in a ischemia-reperfusion induced skin lesion model, but not NFkB[42]. The different results may due to several reasons, such as different disease model, different dosage of quercetin and different measurement of NFKB activation. Further studies should discuss the relationship between quercetin and NFKB using liver failure model in vitro and in vivo.

\section{Conclusions}

In this study, we found that JDNW formula could be used to treat multiple liver diseases including liver failure through a network pharmacology approach. Further, we discussed the mechanisms underlying the multiple biological effects of JDNW formula to verify its potential to treat liver failure. We recognized some JDNW formula related biological processes and KEGG signaling pathways involving in liver failure. We also identified a key bioactive ingredient, quercetin, and found quercetin as a potential NFKB inhibitor to treat liver failure. However, further experimental studies on the relationship between JDNW formula, quercetin, and identified signaling pathways are necessary in the future.

\section{Abbreviations}

JDNW Jieduan Niwan

NFkB Nuclear factor kappa-B

IKK $\beta$ Inhibitor of nuclear factor kappa-B kinase

LPS Lipopolysaccharide

HAS Human serum albumin

D-GalN D-galactosamine

CMAP Connectivity map

PMH Primary mouse hepatocytes

OB Oral bioavailability 
DL Drug likness

BATMAN-TCM Molecular mechANism of Traditional Chinese Medicine

TCMSP Traditional Chinese Medicine System Pharmacology

PU Phyllanthus urinaria

RR Radix rehmanniae

PN Panax Notoginseng

LH Lysimachiae Herba

HMM Hedysarum Multijugum Maxim

VAH Viscum Angulatum Heyne

TKM Trichosanthes Kirilowii Maxim

ALRP Aconiti Lateralis Radix Praeparata

CR Curcumae Rhizoma

RS Radix Salviae

\section{Declarations}

Ethics approval and consent to participate

Not applicable.

Consent for publication

Not applicable.

\section{Availability of data and materials}

The data used to support the findings of this study are available from the corresponding author upon request.

\section{Competing interests}

The authors declare no conflicts of interest.

\section{Funding}


This research was supported by the National Natural Science Foundation of China [Grant no. 81573767].

\section{Authors' contributions}

MCY and DYQ conceived and designed the study; MCY and ZQY wrote the paper; GY, GLY and WXY performed the study and analyzed the data; LJJ and YWL supervised the study and revised the manuscript. All authors read and approved the final manuscript.

\section{Acknowledgements}

Not applicable.

\section{Supplementary Materials}

Supplementary materials contain two tables. Table S1 Detailed information of active compounds in JDNW formula; Table S2 Detailed information of JDNW formula targets. Figure S1 GO terms and KEGG enrichment of JDNW formula related targets.

\section{References}

1. Williams R, Schalm S, O'Grady J. Acute liver failure: redefining the syndromes. The Lancet. 1993;342(8866):273-5.

2. Xiao J, Wang F, Wong N-K, He J, Zhang R, Sun R, Xu Y, Liu Y, Li W, Koike K. Global liver disease burdens and research trends: analysis from a china perspective. Journal of hepatology 2019.

3. Ma Z, Zhang B, Fan Y, Wang M, Kebebe D, Li J, Liu Z. Traditional Chinese medicine combined with hepatic targeted drug delivery systems: A new strategy for the treatment of liver diseases. Biomed Pharmacother. 2019;117:109128.

4. Hou W-x, Hao Y-I, Yang W-I, Tian T, Jiang T-y, Zhang Q-y, Gao L-y. DU Y-q: Effects of Jieduan Niwan Prescription on IL- 6 and TNF- $a$ in Serum and Hepatic Tissue of Acute-on-Chronic Liver Failure Rats. Chinese Journal of Information on Traditional Chinese Medicine. 2018;25(7):49-52.

5. Jinxia L, Lingyun M, Zhang Q, Chen Y, Gao L, Yuqiong D. Effects ofJieduan Niwan Formula on Mitochondrial Apoptotic Pathway in Acute-on-Chronic Liver Failure Rats. Chinese Journal of Information on Traditional Chinese Medicine. 2016;23(4):45-8.

6. CUI L-j DANG, Z-f ZHANG, Q-y XING, J-I CHENY. Effects of the Prescription of Truncation and Inverse Draft on the TNF- $a$, IL-1 $\beta$ and IL- 6 of Serum in Acute-on-chronic Liver Failure Model Rats [J]. Guiding Journal of Traditional Chinese Medicine and Pharmacy 2013, 1.

7. Tan A, Huang H, Zhang P, Li S. Network-based cancer precision medicine: A new emerging paradigm. Cancer letters. 2019;458:39-45.

8. Song X, Zhang Y, Dai E, Du H, Wang L. Mechanism of action of celastrol against rheumatoid arthritis: A network pharmacology analysis. Int Immunopharmacol. 2019;74:105725. 
9. Ding M, Ma W, Wang X, Chen S, Zou S, Wei J, Yang Y, Li J, Yang X, Wang H. A network pharmacology integrated pharmacokinetics strategy for uncovering pharmacological mechanism of compounds absorbed into the blood of Dan-Lou tablet on coronary heart disease. J Ethnopharmacol. 2019;242:112055.

10. Tao Y, Tian K, Chen J, Tan D, Liu Y, Xiong Y, Chen Z, Tian Y: Network Pharmacology-Based Prediction of the Active Compounds, Potential Targets, and Signaling Pathways Involved in Danshiliuhao Granule for Treatment of Liver Fibrosis. Evidence-Based Complementary and Alternative Medicine 2019, 2019.

11. Li S, Qian Y, Xie R, Li Y, Jia Z, Zhang Z, Huang R, Tuo L, Quan Y, Yu Z. Protective effects of a combination of ShengMai-Yin and Ganmaidazao decoction in a mouse model of type 2 diabetes with nonalcoholic fatty liver disease. Journal of Ethnopharmacology 2019:112029.

12. Li F, Dong Y-Z, Zhang D, Zhang X-M, Lin Z-J, Zhang B. Molecular mechanisms involved in druginduced liver injury caused by urate-lowering Chinese herbs: A network pharmacology study and biology experiments. PloS one. 2019;14(5):e0216948.

13. Liu Z, Guo F, Wang Y, Li C, Zhang X, Li H, Diao L, Gu J, Wang W, Li D. BATMAN-TCM: a bioinformatics analysis tool for molecular mechANism of traditional Chinese medicine. Scientific reports. 2016;6:21146.

14. Ru J, Li P, Wang J, Zhou W, Li B, Huang C, Li P, Guo Z, Tao W, Yang Y. TCMSP: a database of systems pharmacology for drug discovery from herbal medicines. J Cheminform. 2014;6(1):13.

15. Piñero J, Bravo À, Queralt-Rosinach N, Gutiérrez-Sacristán A, Deu-Pons J, Centeno E, García-García J, Sanz F, Furlong LI. DisGeNET: a comprehensive platform integrating information on human diseaseassociated genes and variants. Nucleic acids research 2016:gkw943.

16. Zhou Y, Zhou B, Pache L, Chang M, Khodabakhshi AH, Tanaseichuk O, Benner C, Chanda SK. Metascape provides a biologist-oriented resource for the analysis of systems-level datasets. Nature communications. 2019;10(1):1523.

17. Subramanian A, Narayan R, Corsello SM, Peck DD, Natoli TE, Lu X, Gould J, Davis JF, Tubelli AA, Asiedu JK. A next generation connectivity map: L1000 platform and the first 1,000,000 profiles. Cell. 2017;171(6):1437-52. e1417.

18. Wang Z, Sun H, Yao X, Li D, Xu L, Li Y, Tian S, Hou T. Comprehensive evaluation of ten docking programs on a diverse set of protein-ligand complexes: the prediction accuracy of sampling power and scoring power. Physical Chemistry Chemical Physics. 2016;18(18):12964-75.

19. Geethangili M, Ding S-T. A Review of the Phytochemistry and Pharmacology of Phyllanthus urinaria L. Frontiers in pharmacology 2018, 9.

20. Yang W, Hao Y, Hou W, Fang X, Fang P, Jiang T, Ma C, Zhang Q. Jieduan-Niwan Formula Reduces Liver Apoptosis in a Rat Model of Acute-on-Chronic Liver Failure by Regulating the E2F1-Mediated Intrinsic Apoptosis Pathway. Evidence-based complementary alternative medicine: eCAM. 2019;2019:8108503. 
21. Antwi AO, Obiri DD, Osafo N, Forkuo AD, Essel LB. Stigmasterol inhibits lipopolysaccharide-induced innate immune responses in murine models. Int Immunopharmacol. 2017;53:105-13.

22. Aguilar-Guadarrama A, Rios M. Flavonoids, sterols and lignans from Cochlospermum vitifolium and their relationship with its liver activity. Molecules. 2018;23(8):1952.

23. Yin Y, Liu X, Liu J, Cai E, Zhu H, Li H, Zhang L, Li P, Zhao Y. Beta-sitosterol and its derivatives repress lipopolysaccharide/d-galactosamine-induced acute hepatic injury by inhibiting the oxidation and inflammation in mice. Bioorg Med Chem Lett. 2018;28(9):1525-33.

24. Lekić N, Canová NK, Hoř́nek A, Farghali $H$. The involvement of heme oxygenase 1 but not nitric oxide synthase 2 in a hepatoprotective action of quercetin in lipopolysaccharide-induced hepatotoxicity of D-galactosamine sensitized rats. Fitoterapia. 2013;87:20-6.

25. Kemelo MK, Canová NK, Horinek A, Farghali H. Sirtuin-activating compounds (STACs) alleviate Dgalactosamine/lipopolysaccharide-induced hepatotoxicity in rats: involvement of sirtuin 1 and heme oxygenase 1. Physiological research 2017, 66(3).

26. Lu NT, Crespi CM, Liu NM, Vu JQ, Ahmadieh Y, Wu S, Lin S, McClune A, Durazo F, Saab S. A phase I dose escalation study demonstrates quercetin safety and explores potential for bioflavonoid antivirals in patients with chronic hepatitis C. Phytotherapy research. 2016;30(1):160-8.

27. Tai M, Zhang J, Song S, Miao R, Liu S, Pang Q, Wu Q, Liu C. Protective effects of luteolin against acetaminophen-induced acute liver failure in mouse. Int Immunopharmacol. 2015;27(1):164-70.

28. He Y, Xia Z, Yu D, Wang J, Jin L, Huang D, Ye X, Li X, Zhang B. Hepatoprotective effects and structureactivity relationship of five flavonoids against lipopolysaccharide/d-galactosamine induced acute liver failure in mice. Int Immunopharmacol. 2019;68:171-8.

29. Wang W, Guan C, Sun X, Zhao Z, Li J, Fu X, Qiu Y, Huang M, Jin J, Huang Z. Tanshinone IIA protects against acetaminophen-induced hepatotoxicity via activating the Nrf2 pathway. Phytomedicine. 2016;23(6):589-96.

30. Wang H, Chen L, Zhang X, Xu L, Xie B, Shi H, Duan Z, Zhang H, Ren F. Kaempferol protects mice from d-GalN/LPS-induced acute liver failure by regulating the ER stress-Grp78-CHOP signaling pathway. Biomed Pharmacother. 2019;111:468-75.

31. QQ. Y, CX. Z: Chemical studies on the constituents of Phyllanthus urinaria L. Acta pharmaceutica Sinica 1993, 28(11):829-835.

32. Shi J, Shan Y, Zhang Z, Lai J, Li Y. HPLC simultaneous determination of quercetin, kaempferol and biochanin B in Astragalus membranaceus (Fisch.) Bunge. Chin J Pharm Anal. 2010;1:40.

33. Hong $Y$, Chen L. Extraction of quercetin from Herba Lysimachiae by molecularly imprinted-matrix solid phase dispersion. J Chromatogr B Analyt Technol Biomed Life Sci. 2013;941:38-44.

34. Tung BT, Hai NT. Phytochemical and pharmacology effect of Panax notoginseng. Journal of Applied Pharmaceutical Science. 2016;6(08):174-8.

35. Wei Q, Donghai C, Yangyang S, Zhenqiu Z. Determination of Contents of Five Flavonoids in Trichosanthis Pericarpium. Liaoning Journal of Traditional Chinese Medicine. 2018;46(3):591-4. 
36. Luqman S, Pezzuto JM. NFkB: a promising target for natural products in cancer chemoprevention. Phytother Res. 2010;24(7):949-63.

37. Schneider G, Krämer OH. NFKB/p53 crosstalk-a promising new therapeutic target. Biochimica et Biophysica Acta (BBA)-Reviews on Cancer. 2011;1815(1):90-103.

38. Fan H-H, Zhu L-B, Li T, Zhu H, Wang Y-N, Ren X-L, Hu B-L, Huang C-P, Zhu J-H, Zhang X. Hyperoside inhibits lipopolysaccharide-induced inflammatory responses in microglial cells via p38 and NFKB pathways. Int Immunopharmacol. 2017;50:14-21.

39. Tripathi A, Kumar B, Sagi SS. Prophylactic efficacy of Quercetin in ameliorating the hypoxia induced vascular leakage in lungs of rats. PloS one. 2019;14(6):e0219075.

40. Raja SB, Rajendiran V, Kasinathan NK, Amrithalakshmi P, Venkatabalasubramanian S, Murali MR, Devaraj H, Devaraj SN. Differential cytotoxic activity of Quercetin on colonic cancer cells depends on ROS generation through COX-2 expression. Food chemical toxicology. 2017;106:92-106.

41. Fang P, Liang J, Jiang X, Fang X, Wu M, Wei X, Yang W, Hou W, Zhang Q. Quercetin Attenuates dGaLN-Induced L02 Cell Damage by Suppressing Oxidative Stress and Mitochondrial Apoptosis via Inhibition of HMGB1. Front Pharmacol. 2020;11:608.

42. Yin G, Wang Z, Wang Z, Wang X. Topical application of quercetin improves wound healing in pressure ulcer lesions. Exp Dermatol. 2018;27(7):779-86.

\section{Figures}
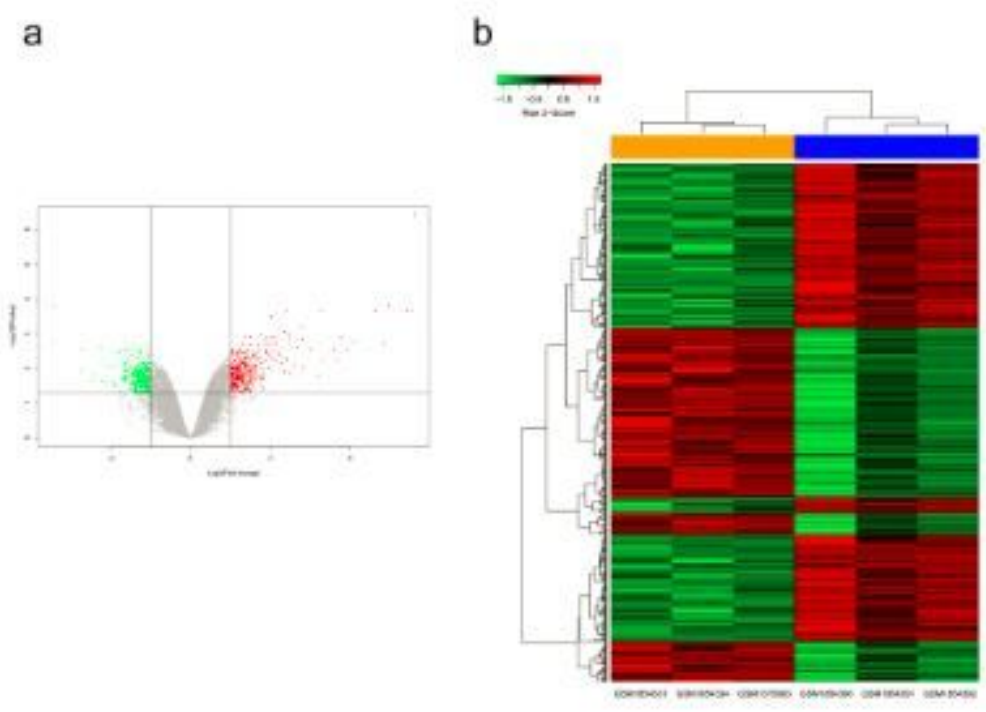

\section{Figure 1}

Ingredient-target and Target-liver disease Network Constructions. (a) Diagram of the ingredient-target interaction network. (b) Diagram of the target-liver disease interaction network. 


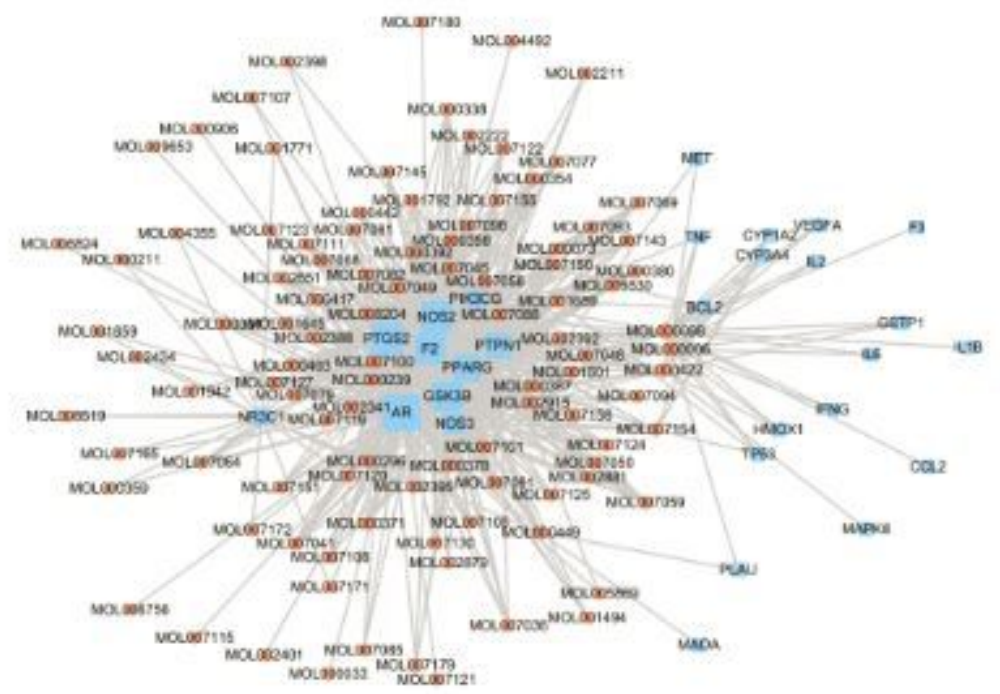

\section{Figure 2}

Ingredient-liver failure related target network.

a

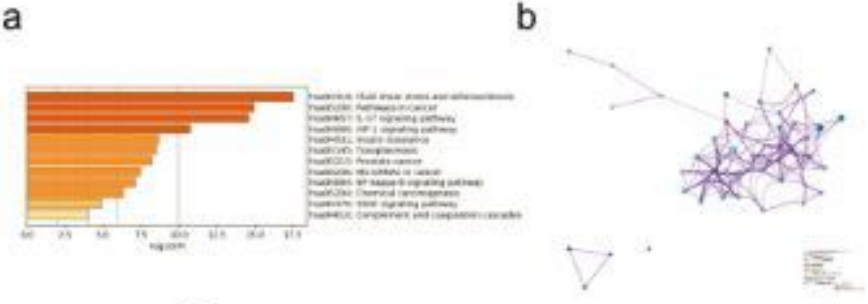

C

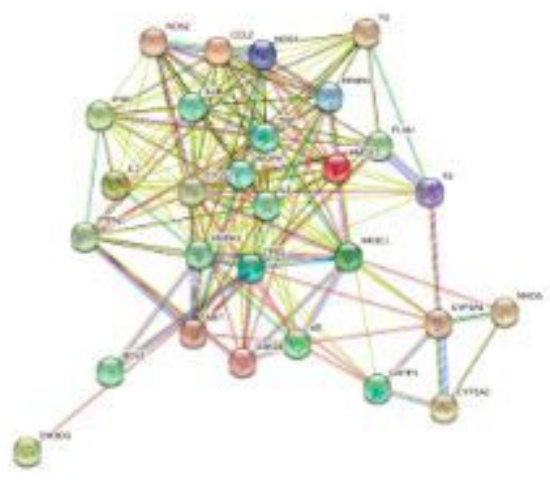

\section{Figure 3}

KEGG pathway enrichment and PPI analysis of JDNW formula against liver failure. (a) Heatmap of Kyoto Encyclopedia of Genes and Genomes (KEGG) enriched terms colored by p-values. (b) Network of KEGG enriched terms. (c) PPI network of JDNW formula against liver failure. 


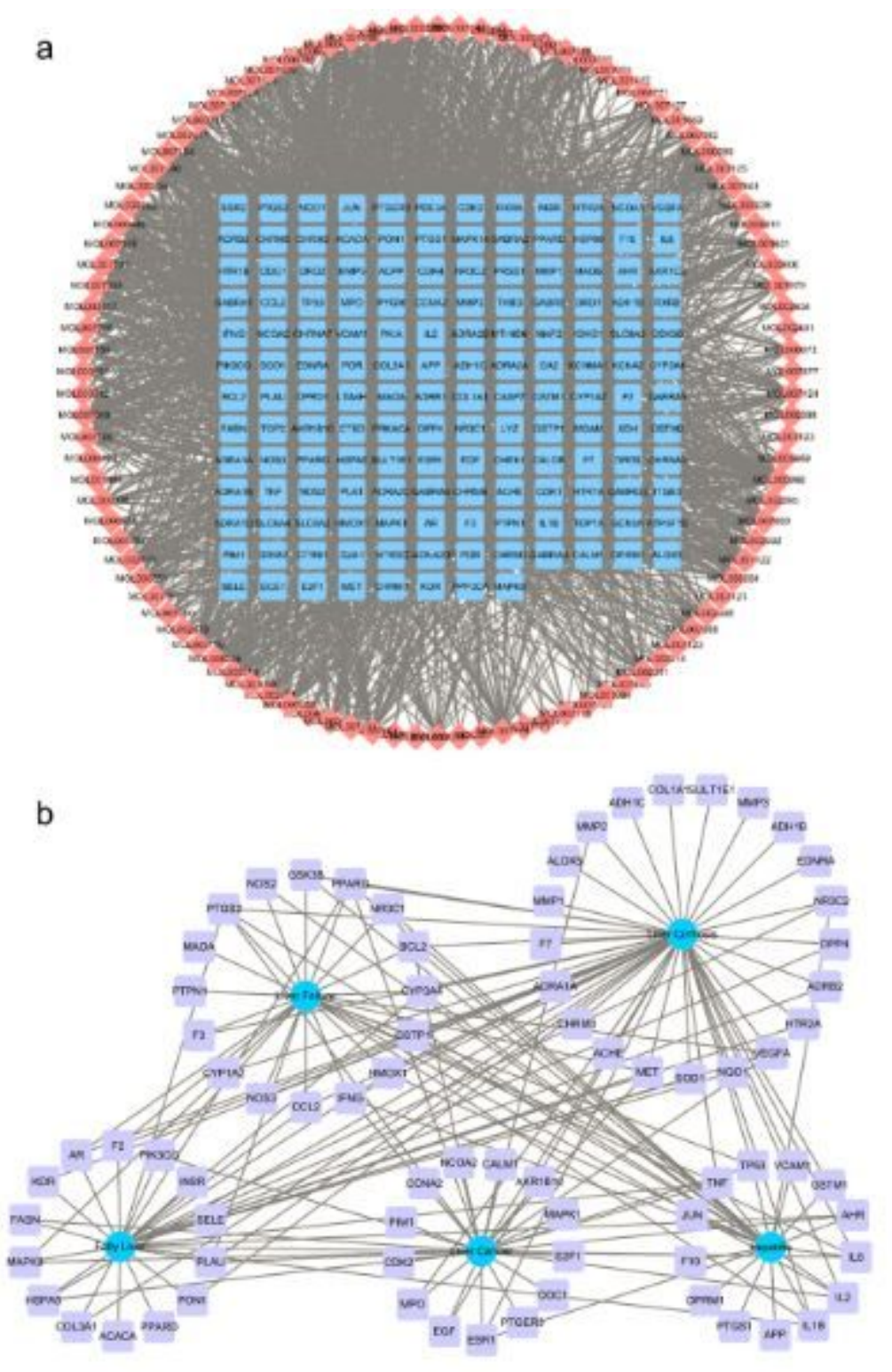

\section{Figure 4}

Identification of different expression genes (DEGs) from GSE72081. (a) Volcano plot of gene expression and (c) heatmap of DEGs by the filtering criteria $(P<0.05$ and $|\log 2 \mathrm{FC}|>1)$. 

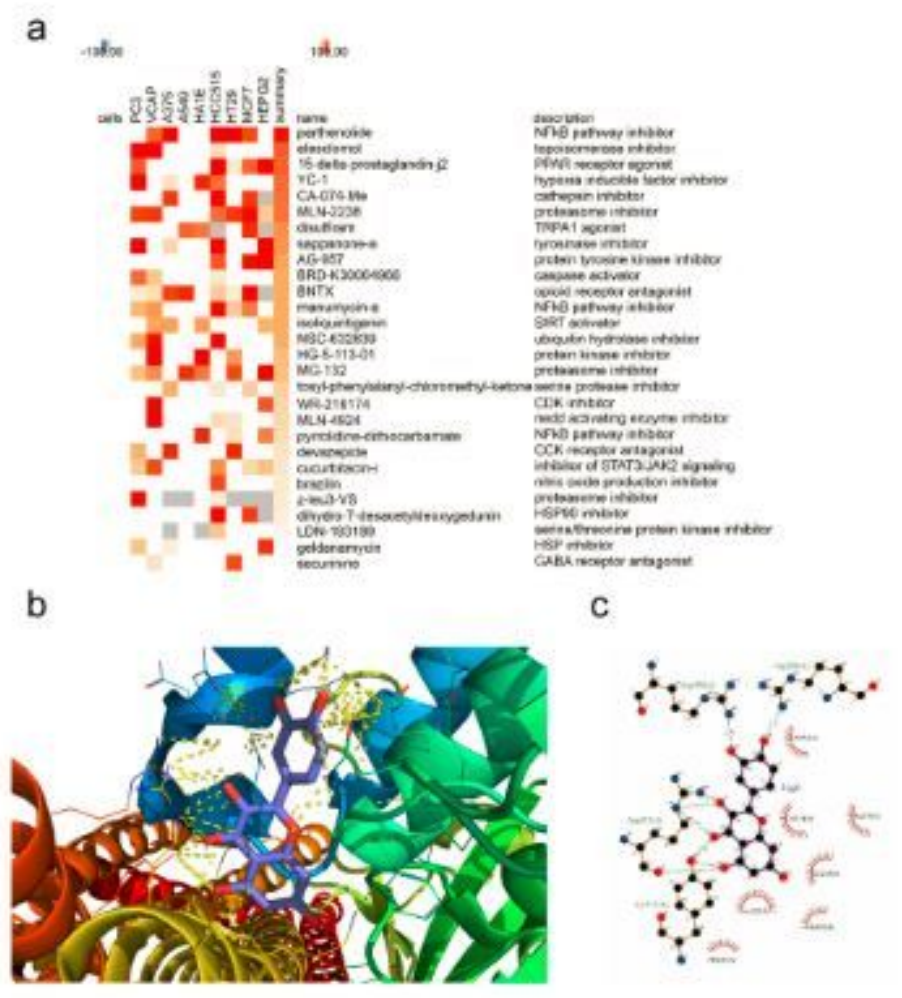

\section{Figure 5}

The relationship between quercetin and NFKB. (a) Heatmap of Top hits after CMAP analysis. (c) Computational analysis of IKK $\beta$ (PDB ID: 4KIK) and quercetin interaction. (c) The residues included in hydrogen bond binding are labeled as green dots, and hydrophobic active site are shown in red.

\section{Supplementary Files}

This is a list of supplementary files associated with this preprint. Click to download.

- StableSfigure.docx 